\title{
Räumliche Konsequenzen des Strukturwandels innerhalb des tertiären Sektors in der Schweiz
}

\section{Einleitung}

Die Schweiz entwickelt sich immer mehr zu einer Dienstleistungsgesellschaft, arbeiten doch heute über die Hälfte aller Erwerbstätigen im tertiären Sektor (vgl. Tabelle 1). Die Dynamik dieses Prozesses hat sich jedoch seit den achtziger Jahren etwas verflacht.

Tab. 1 Entwicklung der 3 Wirtschaftssektoren

\begin{tabular}{|c|c|c|c|}
\hline \multirow{2}{*}{ Jahr } & \multicolumn{3}{|c|}{ Anteile an Erwerbstätigen in Prozenten } \\
& primärer & $\begin{array}{c}\text { sekundärer } \\
\text { Sektor }\end{array}$ & tertiärer \\
\hline 1960 & 14,6 & 46,5 & 38,9 \\
1970 & 8,6 & 46,0 & 45,3 \\
1980 & 7,3 & 39,7 & 53,0 \\
1985 & 6,6 & 38,0 & 55,4 \\
\hline
\end{tabular}

Quelle: BfS: Erwerbstätigenstatistik

Die klassische Begründung dieser Entwicklung beruht auf der These, daß der steigende Wohlstand bei zunehmender Freizeit die Nachfrage nach Dienstleistungen (DL) erhöht. Dieser auf Veränderungen der (End-)Produktestruktur orientierte Erklärungsansatz wird heute vermehrt in Frage gestellt: Der Trend zur Selbstbedienung trägt gegensätzliche Züge, fällt doch einerseits das hierzu notwendige Investitions- oder Konsumgut in der amtlichen Statistik in den sekundären Sektor, andererseits erscheint die Eigenleistung des Konsumenten überhaupt nicht mehr in der Statistik.

Eine weitere Interpretation des DL-Wachstums verweist auf die zunehmende Verlagerung von Tätigkeiten der Haushalte (z. B. Altersvorsorge) oder von privaten Unternehmungen (Transportwesen) auf den Staat und legt hierbei das Schwergewicht auf die Frage nach den Ursachen der steigenden Staatsquote.

Eine dritte These unterstellt demgegenüber nicht eine Zunahme des DL-Konsums, sondern verweist auf den Wandel in der Produktionstechnik (Rationalisierung, mehr DL-Vorleistungen). Hierzu ein Beispiel aus der heutigen Entwicklung im Bankensektor: In diesem werden auf der Konsumentenseite zunehmend Tätigkeiten wegrationalisiert (Selbstbedienungsbanken, Bancomaten usw.), um auf der den Unternehmungen zugewandten Seite (Unternehmensberatung im weitesten Sinne) eingesetzt zu werden.

Infolge des steigenden Vorleistungscharakters von DL verliert der teritäre Sektor auch den Makel, lediglich ein von der Industrie abhängiger Folgesektor zu sein. So bildet heute der Zugang zu spezialisierten DL-Unternehmen eine wesentliche Voraussetzung zur Erhaltung der Konkurrenzfähigkeit von peripheren Industriebetrieben. So etwa bei der Einführung von Innovationen, respektive bei der laufenden Anpassung an neue Marktbedingungen (GERHEUSER 1984).

\section{Strukturwandel innerhalb des tertiären Sektors}

Das starke Wachstum des tertiären Sektors fiel auf Branchenstufe ganz unterschiedlich aus: während einzelne Dienste beschäftigungsmäßig stark zunahmen (z. B. kommerzielle Datenverarbeitung), verzeichneten andere gar deutliche Einbußen (häusliche Dienste in Privathaushalten). Es stellt sich nun die Frage, ob zwischen den stark wachsenden DL-Branchen beziehungsweise zwischen den schrumpfenden Diensten gewisse Gemeinsamkeiten bestehen. So scheint es für das weitere Vorgehen sinnvoll zu sein, in dieses Konglomerat verschiedenster Tätigkeiten eine gewissen Ordnung hineinzubringen. Die gesuchte Typologie sollte folgenden Ansprüchen genügen:

- Die den einzelnen Gruppen zugeordneten Branchen sollten sich bezüglich der Wachstumsdeterminaten möglichst homogen verhalten, und

- es sollte eine bestehende Typologie verwendet werden, um das bereits vorhandene Angebot an Typologien (1) nicht noch um eine neue zu erweitern.

Wir wollen hier die Typologie von Browning/Singlemann (1978) verwenden, da diese einerseits in der neuesten Literatur am häufigsten verwendet wird (GERSHUNY/MILES 1983, HOTZ/WÜRTH 1985, DEISS

Markus Würth, lic. oec. publ., ORL-Institut, ETH Zürich, 8093 Zürich 
Tab. 2 Einordnung der vier DL-Gruppen

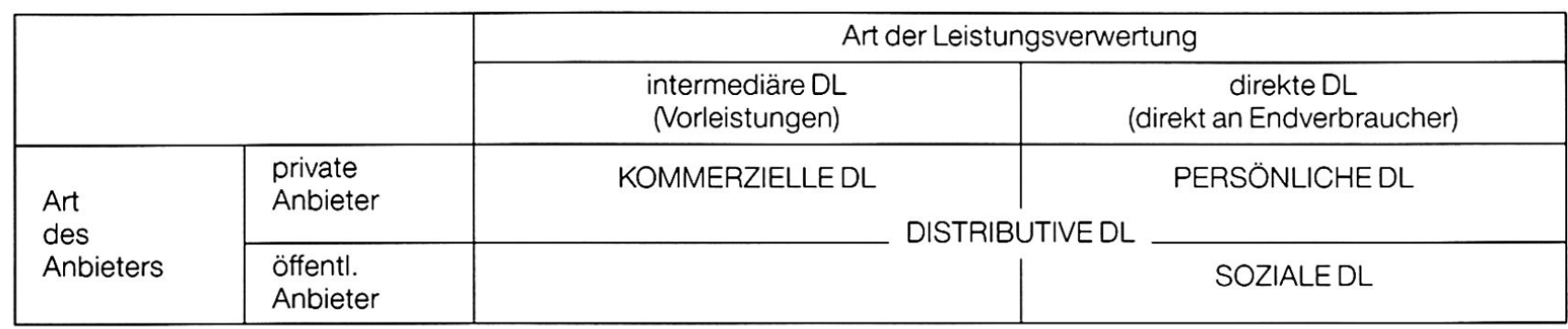

1985, BAILLY/MAILLAT 1986) und andererseits eine umfassende empirische Analyse der Wachstumsdeterminanten des tertiären Sektors für die Schweiz nicht vorliegt.

\subsection{Typologie von Browning-Singlemann}

Browning und Singlemann unterscheiden vier DLGruppen: Kommerzielle DL (Producer Services), distributive DL (Distributive S.), soziale (Social S.) und persönliche DL (Personal S.). Nach den beiden Kriterien "Art der Leistungsverwertung" beziehungsweise "Art des Anbieters» sind diese folgendermaßen einzuordnen (vgl. GERSHUNY/MILES 1983):

Unter die kommerziellen $D L$, welche vorwiegend von privaten Unternehmen angeboten werden und im allgemeinen als Vorprodukte wieder in den Produktionsprozeß einfließen, fallen die Banken, die Versicherungen, die Beratung sowie Immobilien/ Verleih (2).

Persönliche $D L$, welche direkt an den Endverbraucher gelangen, umfassen das Gast- und das Reparaturgewerbe, die Reinigung und die Sonstigen Dienste.

Soziale $D L$, das heißt Dienste, welche vorwiegend von öffentlichen Betrieben angeboten und direkt vom Endverbraucher konsumiert werden, beinhalten die öffentliche Verwaltung (inkl. öffentliches und privates Unterrichtswesen und Forschung), das Gesundheitswesen und die Wohlfahrt (inkl. Kirche, Sport und Kultur).

Distributive $D L$ sind nicht eindeutig in dieses Schema einzuordnen, werden sie doch gerade in der Schweiz zu einem großen Teil auch von öffentlichen Betrieben angeboten und fließen teilweise als Zwischenprodukte wieder in den Produktionsproze $\beta$ ein. Hierunter fallen der Groß- und Kleinhandel, das Post- und Nachrichtenwesen und der Verkehr. Aufgrund von Input-Output-Analysen sowie Untersuchungen bezüglich der Größe des öffentlichen Sektors in der Schweiz scheint die Zuordnung der einzelnen Branchen auf die vier DL-Gruppen durchaus auf die Schweiz übertragbar zu sein (vgl. WÜRTH 1986).

\subsection{Wachstum der DL-Gruppen von 1960 bis 1982}

Betrachtet man die Veränderung der Beschäftigungsanteile innerhalb des tertiären Sektors, so fallen einerseits die großen Anteilsgewinne der sozialen Dienste und der kommerziellen Dienste auf (letztere konnten ihren Anteil um 58 Prozent erhöhen), andererseits die deutlichen Anteilsverluste der persönlichen Dienste (Beschäftigungseinbusse von ca. 30000 Erwerbstätigen).

\subsection{Wachstumsdeterminanten des tertiären Sektors}

In der Literatur wurde dieser Fragestellung bislang wenig Aufmerksamkeit geschenkt. Entweder wurde lediglich der Einfluß eines Faktors (z. B. Haushalteinkommen) untersucht, oder man begnügte sich mit der Formulierung von qualitativen Pauschalaussagen. Schwierigkeiten für eine empirische Untersuchung ergeben sich durch die gegenseitige Abhängigkeit der Faktoren sowie in der Wahl geeigneter Indikatoren (so wird beispielsweise das Einkommen als Indikator für zwei verschiedene Determinanten verwendet).

Fig. 1 Anteilsverschiebungen innerhalb des tertiären Sektors

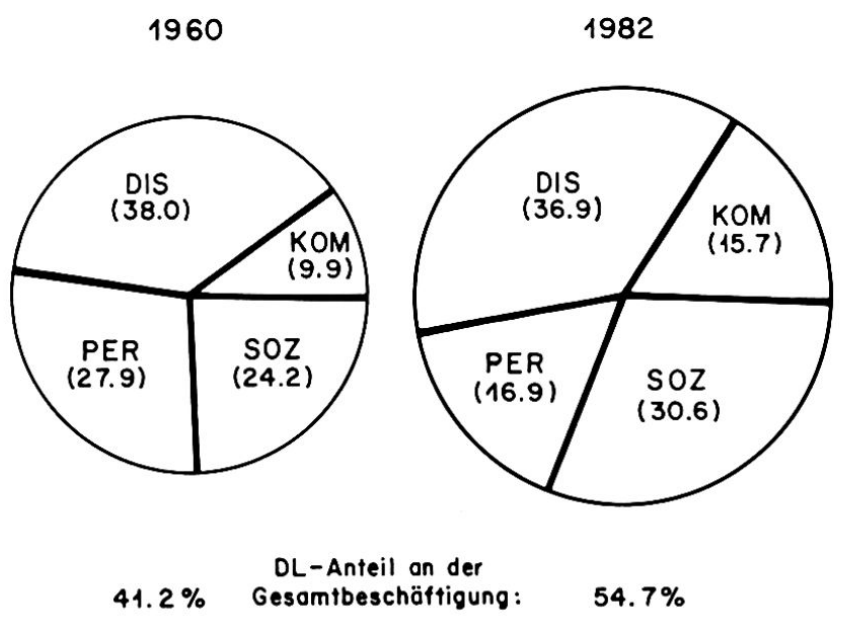

Quelle: Meier (1984) und eigene Berechnungen 
Folgenden Faktoren wird der stärkste Einfluß auf die Beschäftigungsentwicklung des tertiären Sektors zugeschrieben:

\section{A Unterdurchschnittliche Arbeitsproduktivität der DL}

Dadurch schlägt sich eine steigende Nachfrage stärker auf die Beschäftigung nieder als in der übrigen Wirtschaft. Für die Periode 1960 bis 1982 hat diese Differenz jedoch lediglich 0,4 Prozent betragen, wobei die Produktivitätsgewinne im Branchenvergleich erheblich schwankten (Meier 1984).

\section{$B$ überdurchschnittliche Einkommenselastizität $\operatorname{der} D L$}

Bei steigendem verfügbarem Haushalteinkommen nimmt der Anteil des DL-Konsums an den Gesamtausgaben zu, da vermehrt Bedürfnisse höherer Ordnung (kulturelle Bedürfnisse) befriedigt werden können, letztere aber mit wenigen Ausnahmen statistisch dem tertiären Sektor zugeordnet werden.

\section{Substitution von DL durch langlebige Konsumgüter}

Die infolge der unterdurchschnittlichen Arbeitsproduktivitätsentwicklung oft über dem Durchschnitt liegende Preisentwicklung einerseits, als andererseits auch das Auftreten immer preisgünstigerer Substitute (TV, Waschmaschine, Auto usw.) haben einen Rückgang der Nachfrage vor allem nach persönlichen Diensten bewirkt.

\section{Demographische Faktoren}

Als für die Entwicklung des tertiären Sektors am bedeutsamsten wären hier die durchschnittliche Haushaltgröße und der Rentneranteil einer Volkswirtschaft zu erwähnen. Die Zunahme des letzteren scheint vor allem die sozialen DL positiv beeinflußt zu haben (WÜRTH 1986).

Währenddem die bisherigen Faktoren primär für die Entwicklung der direkten DL relevant sind, erklären die folgenden Faktoren die Dynamik der intermediären Dienste.

\section{E Komplementarität der $D L$}

So besteht eine positive Abhängigkeit zwischen intermediären DL und der allgemeinen Wirtschaftsentwicklung, da diese Dienste entweder als Vorleistungen in die Produkte einfließen (Planung, Marktforschung) oder vermehrt gebündelt mit Industrieprodukten angeboten werden (Finanzierung). Die Branchen Beratung, Immobilien/Verleih, Banken und Großhandel weisen denn auch eine über dem schweizerischen Durchschnitt liegende konjunkturelle Abhängigkeit auf (MEIER 1984).
Die Komplementarität hat sich in den letzten Jahren noch verstärkt, da heute infolge des zunehmenden Informationsbedarfes der Wirtschaft immer mehr DL-Tätigkeiten als Vorleistungen in den Produktionsprozeß einfließen. Diese können sowohl unternehmensintern erbracht werden, man spricht in diesem Falle von einer Tertiarisierung des sekundären Sektors, oder extern zugekauft werden. Erst letzteres bewirkt ein Ansteigen des tertiären Sektors. Als weitere Bestimmungsfaktoren können somit der steigende Informationsbedarf und die vermehrte Externalisierung von Diensten (3) voneinander unterschieden werden.

\section{F Zunehmender Informationsbedarf der Wirtschaft}

Ein Großteil intermediärer DL-Tätigkeiten kann man als Informationstätigkeiten bezeichnen. Als Gründe für deren Zunahme werden etwa angeführt:

- Die infolge der gestiegenen Mobilität zunehmende internationale Verflechtung (mehr Marktinformationen), eine damit verschärfte internationale Konkurrenz (Werbung, Marketing) sowie immer kürzere Produktezyklen (Forschung und Entwicklung, Planung).

- Steigende Unternehmensgrößen und zunehmende wirtschaftliche Konzentrationserscheinungen verursachen einen höheren Steuerungsund Kontrollaufwand.

- Die durch die Telematik neu eröffneten Möglichkeiten bei der Übertragung, Verarbeitung und Speicherung von Informationen.

\section{$G E x$ - bzw. Internalisierungsstrategien}

Obwohl auch für die Schweiz eine zunehmende Arbeitsteilung bei steigendem Spezialisierungsgrad der einzelnen Dienste festzustellen ist - stieg doch der Vorleistungsanteil von 49,2 Prozent (1960) auf 53,4 Prozent (1982) an (MEIER 1984) -, steht die Schweiz im internationalen Vergleich noch eher am Anfang einer Entwicklung, welche in den USA (DL-Anteil von 66 Prozent) bereits wesentlich weiter fortgeschritten ist (GROSS 1983). Folgende Faktoren beeinflussen den Entscheid bezüglich der Externalisierung von Diensten (MARSHALL 1982, wIL LIAMSON 1978): die Vertraulichkeit der benötigten Information, die Regelmäßigkeit des DL-Bedürfnisses, das notwendige beziehungsweise betriebsintern vorhandene Qualifikationspotential, die Kosten des externen Bezugs und die Erreichbarkeit der DL-Anbieter.

Eine Ausrichtung der Fragestellung lediglich auf den Externalisierungsentscheid scheint mir wenig sinnvoll zu sein. Es sind deshalb vermehrt auch die Entwicklungen außerhalb des tertiären Sektors (Tertiarisierungsproze $\beta$ in der Industrie) zu untersuchen (4). 


\section{Räumliches Verhalten des tertiären Sektors}

\subsection{Funktionale Standortabhängigkeit}

Es stellt sich nun die Frage, ob die verschiedenen DL-Gruppen ein deutlich unterschiedliches Standortverhalten zeigen und ob bestimmte DL für die Entwicklung einer Region von Bedeutung sind.

Mit Hilfe einer einfachen Korrelationsanalyse, diese mißt die Stärke und Richtung eines linearen Zusammenhangs zweier Variabeln, wobei eine solche funktionale Korrelation noch nichts aussagt über den kausalen Zusammenhang beider Größen, läßt sich tatsächlich ein enger funktionaler Zusammenhang zwischen der Bevölkerungsverteilung und den direkten Diensten beziehungsweise zwischen den intermediären DL und den Wirtschaftsstandorten nachweisen (vgl. Tabelle 3 ).

Tab. 3 Standortabhängigkeiten der $\mathrm{DL}^{*}$ gemessen mittels Korrelationskoeffizient

\begin{tabular}{|l|c|c|c|}
\hline $\begin{array}{l}\text { DL- } \\
\text { Beschäftigte }\end{array}$ & $\begin{array}{c}\text { Ein- } \\
\text { wohner }\end{array}$ & $\begin{array}{c}\text { Hauptsitze } \\
\text { (ungewichtet) }\end{array}$ & $\begin{array}{c}\text { Hauptsitze }^{\star \star} \\
\text { (gewichtet mit } \\
\text { Umsatz) }\end{array}$ \\
\hline $\begin{array}{l}\text { intermediäre DL } \\
\text { direkte DL }\end{array}$ & .91 & .94 & .86 \\
\hline
\end{tabular}

* gemessen über die 106 MS-Regionen (vgl. Schuler/Nef 1983)

** 1000 größte Unternehmungen in der Schweiz ohne Banken und Versicherungen (Schweizerische Handelszeitung 1984)

Räumlich am stärksten konzentriert sind die kommerziellen Dienste: 47 Prozente ihrer Beschäftigten haben ihren Arbeitsplatz in einem der fünf Großstadtzentren. Am dispersesten verteilt sind die persönlichen DL (HOTZ-HART/WÜRTH 1985).

Die für den DL-Sektor klassische Standorttheorie von Christaller vermag lediglich das Standortverhalten einiger direkter Dienste zu erklären. Solche an den Transportkosten orientierten Ansätze verlieren infolge der zunehmenden Mobilität der Bevölkerung zusehends an Aussagekraft. Für kommerzielle Dienste, welche vorwiegend Informationstätigkeiten beinhalten, prägen vor allem Informationsflüsse ihre Beziehungen zur Umwelt. So bildet die Zugänglichkeit relevanter Informationen ein wesentliches Standortkriterium dieser Dienste (BAHRS-DISCHER 1981).

Die steigende arbeitsteilige Verflechtung zwischen kommerziellen DL und DL-Tätigkeiten in der Industrie erhöht infolge zunehmender Spezialisierung deren gegenseitige Abhängigkeit und somit die Häufigkeit des Informationsaustausches. Diese funktionalen Abhängigkeiten bewirken in der Folge auch räumliche Clusters (5). Es ist an dieser Stelle jedoch daran zu erinnern, daß zwischen der Standortwahl und dem Externalisierungsentscheid gegenseitige Abhängigkeiten bestehen (vgl. Abschnitt 2.3).

Die entstehenden Kommunikationszentren bedingen, daß auch die für die Infrastruktur der Kommunikationsbeziehungen notwendigen Dienste wie das Nachrichtenwesen, die Datenverarbeitung, Flughäfen usw. in der Nähe dieser Zentren lokalisiert sind.

Es stellt sich somit die Frage, ob solche räumlichen Clusters auch in der Schweiz zu beobachten sind. Da keine Zahlen bezüglich der Kommunikationskontakte von Unternehmen vorliegen, wollen wir untersuchen, ob eine deutlich höhere Korrelation zwischen dem Anteil von DL-Tätigkeiten in einer Region und der relativen Präsenz von kommerziellen Diensten gegenüber andern DL-Gruppen festzustellen ist.

Stellt man nun die Arbeitsplätze der jeweiligen DLGruppe, entnommen aus der Volkszählung 1980, dem Anteil «nicht-produktiver Tätigkeiten» in der Industrie gemäß Industriestatistik 1981 gegenüber (GEILINGER 1984), so zeigt sich bei allgemein kleinen Korrelationswerten doch ein deutlich überdurchschnittlicher Zusammenhang zu den kommerziellen DL bzw. ein negativer Zusammenhang zur räumlichen Präsenz von persönlichen Diensten (vgl. Tabelle 4).

Der Koeffizient bei den kommerziellen DL erhöht sich jedoch deutlich bei Nichtberücksichtigung der beiden Ausreißer-Regionen Glattal (hohe Präsenz von Hauptsitzen beziehungsweise DL-Tätigkeiten in der Industrie bei schwacher Präsenz von kommerziellen DL) und Lugano (umgekehrt zum Glatttal).

Diese Werte bestätigen, daß zwischen den Standorten beider Tätigkeiten nicht eine Entweder/OderBeziehung besteht (Internalisierung beziehungsweise Externalisierung von DL), sondern daß beide aufeinander oder zumindest auf dieselben Standortfaktoren (Milieu) angewiesen sind.

Verwendet man anstelle des Anteils «nicht-produktiver Tätigkeiten» die Anzahl Hauptsitze der 1000 größten Unternehmungen in der Schweiz, so ändert sich das Bild nur insofern, als sich zusätzlich zu den kommerziellen DL auch für die distributiven DL höhere Korrelationswerte ergeben. Bei Berücksichtigung des jeweils erzielten Umsatzes einer Unternehmung schwächt sich dieser Wert jedoch wieder ab. Diese Zusammenhänge sind für die räumliche Entwicklung von Bedeutung, da Hauptsitze im allgemeinen nicht nur über einen überdurchschnittlich hohen Anteil an DL-Tätigkeiten verfügen, sondern in vielen Fällen auch über den externen Bezug von DL - auch für ihre Filialen - entscheiden. 
Tab. 4 Funktionale Standortabhängigkeiten der Dienste

\begin{tabular}{|c|c|c|c|c|}
\hline Branchen & \multicolumn{4}{|c|}{ (Arbeitsplätze gemäß VZ 80) } \\
\hline Tätigkeiten & KOM & DIS & $\mathrm{SOZ}$ & PER \\
\hline $\begin{array}{l}\text { (1) Anteil «nicht-pro- } \\
\text { duktiver Arbeitsplätze» } \\
\text { in der Industrie } 1981\end{array}$ & $\begin{array}{c}.389 \\
{[.473]}\end{array}$ & $\begin{array}{l}.205 \\
{[.250]}\end{array}$ & $\begin{array}{c}.223 \\
{[.238]}\end{array}$ & $\begin{array}{l}-.440 \\
{[-.424]}\end{array}$ \\
\hline $\begin{array}{l}\text { (2) Anzahl Hauptsitze } \\
\text { der } 1000 \text { grössten } \\
\text { Unternehmen in der } \\
\text { Schweiz (ohne Banken, } \\
\text { Versicherungen) }\end{array}$ & $\begin{array}{c}.551 \\
{[.636]}\end{array}$ & $\begin{array}{c}.436 \\
{[.387]}\end{array}$ & $\begin{array}{c}.186 \\
{[.216]}\end{array}$ & $\begin{array}{r}-.199 \\
{[-.192]}\end{array}$ \\
\hline $\begin{array}{l}\text { (3) wie (2), aber } \\
\text { lediglich Industrie- } \\
\text { unternehmen }\end{array}$ & $\begin{array}{c}.541 \\
{[.632]}\end{array}$ & $\begin{array}{c}.456 \\
{[.400]}\end{array}$ & $\begin{array}{c}.198 \\
{[.232]}\end{array}$ & $\begin{array}{l}-.254 \\
{[-.249]}\end{array}$ \\
\hline $\begin{array}{l}\text { (4) wie (2), aber } \\
\text { gewichtet mit ihrem } \\
\text { jeweiligen Umsatz }\end{array}$ & $\begin{array}{c}.559 \\
{[.633]}\end{array}$ & $\begin{array}{c}.349 \\
{[.364]}\end{array}$ & $\begin{array}{l}.194 \\
{[.203]}\end{array}$ & $\begin{array}{l}-.153 \\
{[-.150]}\end{array}$ \\
\hline
\end{tabular}

[ ] ohne die MS-Regionen "Glattal" und "Lugano" Quellen: (1) Geilinger (1984)

(2-4) Schweizerische Handelszeitung (1984)

Branchen: BfS, Informationsraster

\subsection{Weitere Charakteristiken kontaktintensiver DL}

Sowohl «nicht-produktive Tätigkeiten» in der Industrie als auch kommerzielle Dienste weisen einen deutlich höheren Anteil an qualifiziertem Personal auf als der Branchendurchschnitt in der Schweiz (GEILINGER 1984); hinzu kommt, daß die kommerziellen DL sehr arbeitsintensiv produzieren. Beide Tätigkeiten sind somit auf einen qualifizierten Arbeitsmarkt angewiesen.

Für die Raumentwicklung wohl ebenso bedeutsam ist die Tatsache, daß diese Dienste aufgrund ihrer relativ hohen Wertschöpfung und ihres geringen Raumbedarfes (hohe Wertschöpfung pro Quadratmeter) auch in der Lage sind, ihre Standortwünsche durchzusetzen. So erzielen sie im Branchenvergleich die größte Bodenrente (HOTZ-HART/WÜRTH 1985).

\section{Raumwirtschaftliche Konsequenzen des Strukturwandels}

Die Dynamik des Strukturwandels, das heißt der rasch wachsende Anteil kontaktintensiver Dienste im tertiären als auch im sekundären Sektor, wird durch ihre funktionale Standortabhängigkeit auch auf die Raumentwicklung übertragen: es entsteht ein zirkulär-kumulativer Proze $\beta$, welcher zur Ausbildung einiger weniger dominanten Kommunikations- und Entscheidungszentren führt. Als konstituierende Elemente dieses Prozesses wären zu nennen:

1. Die am stärksten wachsenden Tätigkeiten sind auch am stärksten auf die Zentren ausgerichtet. Dies dürfte auch in Zukunft die Nachfrage nach zentralen Standorten verstärken.

2. Bei beschränkten Baulandreserven in diesen Zentren verstärkt sich das Bodenpreisgefälle zwischen Zentren und Peripherie. Ohne flankierende politische Maßnahmen werden somit auch weiter Funktionen wie Wohnen, Industrieproduktion, persönliche DL usw. infolge ihrer tieferen Bodenrente aus den Zentren verdrängt. Ebenso wird hierdurch eine räumlich-funktionale Arbeitsteilung innerhalb von Mehr-Betriebs-Unternehmen gefördert.

3. Eine zunehmende wirtschaftliche Konzentration verstärkt diesen Prozeß, da «durch die Übernahme peripherer Betriebe durch Großunternehmen in signifikanter Weise qualifizierte Arbeitsplätze im dispositiven Bereich verloren gehen» (MÜlleR 1980). Hierdurch treten die übernommenen Betriebe auch Kompetenzen in bezug auf die Auftragserteilung an spezialisierte DL-Betriebe ab.

4. Neuerungen in der Telematik mögen diesen Prozeß sowohl in verstärkender als auch in abschwächender Weise zu beeinflussen. Einerseits verstärken sie diesen Prozeß, als sich durch deren Einsatz Möglichkeiten einer zentralen Steuerung und Kontrolle eröffnen, welche die Wahrscheinlichkeit einer unternehmensinternen Standortspaltung bei gleichzeitiger Zentralisierung von Entscheidungskompetenzen erhöht. Abschwächend wirken sie, als sich hierdurch ein großes Rationalisierungspotential an Informationstätigkeiten eröffnet und eine kleinräumige Dekonzentration - infolge des zunehmenden Bodenpreisgefälles von weniger auf persönliche Kontakte angewiesenen DL-Tätigkeiten als durchaus möglich erscheint.

$\mathrm{Da} \beta$ also eine Dekonzentration von Bevölkerung und Wirtschaft durchaus von einer zunehmenden Hierarchisierung des Raumes, insbesondere von einer verstärkten Zentralisierung von Entscheidungsmacht begleitet sein kann, scheint somit für die Schweiz durchaus plausibel zu sein. Hierbei spielt der tertiäre Sektor eine wichtige Rolle, wobei jedoch die herkömmliche statistische 3-Sektoren-Gliederung den heute bestehenden funktionalen Abhängigkeiten der verschiedenen Branchen beziehungsweise Tätigkeiten nicht mehr genügt.

Empirisch fundierte Aussagen über diese Zusammenhänge liegen zurzeit jedoch für die Schweiz noch kaum vor. Dies mag mit dazu beitragen, da $\beta$ bis anhin mit Ausnahme des Gastgewerbes - im Gegensatz zu einigen Nachbarländern (vgl. WÜRTH 1986) - DL in der schweizerischen Regionalpolitik noch kaum in den Katalog förderungswürdiger Branchen aufgenommen worden sind. 
Umgekehrt sind bereits Instrumente in Kraft, welche die weitere räumliche Konzentration von DL in den Zentren zu beschränken versuchen (bspw. Wohnanteilsplan beziehungsweise Wohnerhaltungs-Gesetz in der Stadt Zürich). Hierbei handelt es sich jedoch vorwiegend um Maßnahmen, welche die kleinräumige Verdrängung der Wohnnutzung zu verhindern suchen.

Die Tatsache, daß die für die Entwicklung einer Region bedeutsamen Dienste - wie ein Großteil der kommerziellen Dienste - auf spezifisch in den Zentren vorhandene Standortfaktoren angewiesen sind (beschränktes Mobilitätspotential), läßt eine staatliche Lenkungspolitik mittels finanzieller Anreize als nur beschränkt wirksam erscheinen. Im Rahmen einer als Bestandespolitik zu verstehenden, vermehrt dienstleistungsorientierten Regionalpolitik sind nun kommerzielle DL in der staatlichen Förderungspraxis den Industriebranchen gleichzustellen: In peripheren Regionen ansässige spezialisierte DL-Betriebe sollten somit aus regionalpolitischen Gründen vermehrt finanziell unterstützt werden.

\section{Anmerkungen:}

1 Eine Übersicht über die gebräuchlichsten Typologien vermittelt Barcet (1986).

2 Die hier verwendete Branchenabgrenzung entspricht jener von Meier (1984).

3 Unter einer Externalisierung von DL im Industriebereich werden hier Vorleistungsbezüge einer Industrieunternehmung von einer Unternehmung verstanden, deren Haupttätigkeit statistisch in den teritären Sektor fällt.

${ }^{4}$ So wird die gegenwärtige statistische Abgrenzung der drei Sektoren der heutigen Situation nicht mehr gerecht. Es werden denn auch laufend neue Typologien vorgeschlagen (C.E.A.T. 1985, Barcet 1986).

5 Goddard (1973) hat für London festgestellt, daß Bürokomplexe, das heißt Banken, Werbe-, Handelsgesellschaften usw., die arbeitsteilig miteinander verflochten sind, auch standörtliche Konglomerate bilden.

${ }^{6}$ Aus datentechnischen Gründen (Informationsraster) umfassen die kommerziellen $\mathrm{DL}$ auch einige persönlichen und sozialen DL. Ohne diese dürften die Differenzen noch deutlicher zutage treten.

\section{Literaturverzeichnis}

BAHRS-DISCHER, E. (1981): Kommunikativbedingte Wirtschaftsstandorte, Arbeitshefte des Instituts für Stadt- und Regionalplanung der technischen Universität Berlin, Bd. 20
BAILLY, A. S./MAILLAT, D. (1986): Le secteur tertiaire en question, activités de service, développement économique et spatial, Paris

BARCET, A. (1986): Les services dans le système productif vers une typologie de la production de services, unveröff. Manuskript im Rahmen des Kolloquiums "Les services et le développement économique: nécessité et utilité d'une nouvelle typologie des activités de services" in Genf

Bfs (Bundesamt für Statistik): Informationsraster, Volkszählung 1980, Erwerbstätigenstatistik

BROWNING, H. C./SINGLEMANN, J. (1978): The Transformation of the US Labour Force: The Interaction of Industry and Occupation. In: Politics and Society, 8 (7-4). Zitiert nach Gershuny (1983)

C.E.A.T. (1985): Nouvelle nomenclature du système de production et rôle des activités de services, Lausanne

DEISS, J. (1985): Diagnostic et perspectives du tertiaire fribourgeois. In: VALARCHÉ, J. et al. (Hrsg.): Le secteur tertiaire et le nouveau développement régional, Fribourg

GEILINGER, U. (1984): Ausmaß, Ursachen und Folgen der funktionalen Arbeitsteilung zwischen Regionen in der schweizerischen Wirtschaft, Diss., Zürich

GERHEUSER, F. (1984): Periphere Mittelzentren als Entwicklungszentren (unveröffentl. Manuskript), Windisch

GERSHUNY, J. I./MILES, I. D. (1983): The New Service Economy, London

GODDARD, J. B. (1973): Office Linkages and Location, Oxford

GROSS, P. (1983): Die Verheißungen der Dienstleistungsgesellschaft: soziale Befreiung oder Sozialherrschaft?, Opladen

HOTZ-HART, B.WÜRTH, M. (1985): Strukturwandel im Dienstleistungssektor und Stadtentwicklung, in: DISP Nr. 80/81, ETH, Zürich

MARSHALL, J. N. (1982): Linkages between manufacturing industry and business services. In: Environment and Planning $A$, vol. 14

MEIER, P. (1984): Daten für den tertiären Sektor in der Schweiz - Wertschöpfung, Beschäftigung und Preise von 1960-1982 für 18 Branchen, Forschungsbericht Nr. 10 des NFP "Wirtschaftsentwicklung", St. Gallen

MÜLLER, K. (1980): Auswirkungen allgemeiner Unternehmenskooperationen. In: Informationsbulletin des NFP “Regionalprobleme" Nr. 7. Zitiert nach: OLBRICH, J. (1984): Regionale Strukturpolitik mit Büroarbeitsplätzen? In: Raumforschung und Raumordnung, Heft 4-5

SCHULER, M./NEF, R. (1983): Räumliche Typologien des schweizerischen Zentren-Peripherien-Musters, Arbeitsbericht Nr. 35 des NFP "Regionalprobleme», Bern

Schweizerische Handelszeitung (1984): SHZ-Liste 1984: Die größten Unternehmen in der Schweiz

WILLIAMSON, O. E. (1978): Market and Hierarchies: Analysis and Antitrust Implications, a Study of the Economics of Internal Organization, London

WÜRTH, M. (1986): Dynamik des tertiären Sektors und Raumentwicklung, Bericht zur Orts-, Regional- und Landesplanung Nr. 59, ETH, Zürich 